Proposition d'article pour la revue

Économie et Prévision

\title{
Un modèle de carrières types dynamiques pondérées pour le Régime général d'assurance vieillesse - Une application aux conséquences de la réforme de 2003
}

\author{
Karine BRIARD ${ }^{1}$ \\ CNAV - Direction de l'Actuariat Statistique \\ CERPEM - Université Paris IX Dauphine
}

Les trajectoires suivies par les assurés du Régime général sont diverses et cette hétérogénéité rend difficile l'appréciation de la situation des futurs retraités. Un modèle de projection structuré autour de carrières représentatives et pondérées permet d'appréhender les mécanismes entrant en jeu et les disparités intra- et intergénérationnelles qu'ils induisent.

Une illustration des potentialités d'un tel modèle nous est donnée par l'examen des effets de la réforme des retraites de 2003, laquelle fixe les modalités d'une évolution continue des paramètres de calcul des pensions et modifie parallèlement les règles applicables à certaines catégories d'assurés.

Codes JEL : J11, J26, H55

Mots-Clés : réforme des retraites, secteur privé, carrières types, modèles de projection

\footnotetext{
${ }^{1}$ CNAV - Direction de l'Actuariat Statistique, 110 avenue de Flandre 75951 PARIS Cedex 19, Tel: 01.55.45.62.14, Fax: 01.55.45.51.83, E-mail : karine.briard@.cnav.fr.
} 
En France, les salariés du secteur privé sont affiliés au Régime général de la Sécurité Sociale dont les prestations retraite sont gérées par la Caisse Nationale d'Assurance Vieillesse (Cnav). Cette population présente des parcours professionnels très hétérogènes, parfois discontinus, marqués par le chômage et, alors que certains travailleurs ne font que transiter par le secteur privé, d'autres y effectuent toute leur carrière. Cette diversité constitue une difficulté majeure pour estimer les charges qu'aura à assumer le régime dans les années à venir. En effet, le montant servi à la retraite est très sensible au profil de la carrière suivie et la modification des règles de calcul conséquente à la dernière réforme, intervenue en 2003, ne fait que renforcer l'incertitude sur l'évolution future de la pension versée en moyenne à chaque retraité.

Les modélisations pèchent souvent par leur caractère réducteur ou, au contraire, par un souci pointilliste de reproduire la diversité individuelle au risque de se détourner de leur finalité. Une voie intermédiaire doit donc être recherchée et un modèle structuré autour d'un nombre limité de parcours professionnels représentatifs paraît répondre à l'ambition d'anticiper les disparités intra- et intergénérationnelles au moment de la retraite.

Cet article présente les conséquences de la réforme de 2003 sur l'évolution de la pension des retraités du Régime général. Il illustre, dans le même temps, les atouts d'un modèle de carrières types dynamiques pour appréhender, selon le parcours professionnel suivi, la portée des différentes règles de calcul des pensions et les conséquences de leur modification. Dans la première partie, l'article décrit les modalités de calcul au Régime général et expose les grandes lignes de la réforme de 2003 relatives aux droits propres contributifs. Une deuxième partie présente les caractéristiques, les atouts et les limites des méthodes de projection actuellement utilisées pour l'évaluation de la politique en matière de retraite. Dans la troisième partie, un nouvel outil est proposé pour le Régime général : le modèle de carrières types dynamiques et pondérées Eclipss. Enfin, la quatrième partie présente les projections d'évolution de la pension attribuée à la liquidation en adoptant successivement approches normative et positive de façon à éclairer sur les objectifs et les conséquences de la réforme de 2003.

\section{Le calcul de la pension au régime général}

\section{Principe général}

La pension servie par la Cnav ne peut excéder $50 \%$ du plafond de la Sécurité Sociale $(30192 €$ au 01/01/2005), les régimes Arrco et Agirc assurant alors un complément calculé sur une base contributive.

Les prestations du Régime général sont définies en annuités, la pension est calculée comme une fraction du salaire annuel moyen (Sam) selon un taux qui dépend de la durée d'activité. Outre les périodes de salariat qui sont soumises à cotisations, les épisodes de chômage, de maladie, d'invalidité, pour lesquels l'assuré est indemnisé par le système de protection sociale, permettent également de valider « à titre gratuit » des trimestres d'assurance appelés « périodes assimilées ».

En notant $D_{R G}, D_{T R}$ et $\delta$ les paramètres de calcul des pensions, respectivement la durée d'assurance rémunérée par le Régime général, la durée totale (tous régimes) requise pour le taux plein et la décote sur le taux par trimestre manquant, et $d_{R G}, d_{T R}, A$ les paramètres individuels, respectivement les durées d'assurances validées au Régime général et tous régimes et l'âge au départ en retraite, le montant de base de la pension $P$ se calcule selon l'expression simplifiée suivante :

$$
\begin{gathered}
P=\operatorname{Sam} \times \min \left(1, d_{R G} / D_{R G}\right) \times \tau \\
\text { avec } \tau=50 \%-\delta \times \min \left(4 \times(65-A), \max \left(0, D_{T R}-d_{T R}\right)\right)
\end{gathered}
$$

Si le taux plein est accordé ( $\tau=50 \%$ ), le montant $P$ est comparé au minimum contributif qui, s'il lui est supérieur, sera servi au prorata de la durée d'assurance validée au Régime général. 


\section{Le calcul de la pension suite à la réforme de 2003}

Dans son article 5, la loi du 21 août 2003 portant réforme affiche l'objectif d'une stabilisation du ratio entre temps de travail et temps de retraite par une règle de partage des gains d'espérance de vie $(2 / 3$ travail $-1 / 3$ retraite). Elle instaure des mécanismes d'ajustement semi-automatique des paramètres de calcul des pensions aux évolutions démographiques et prolonge ainsi la réforme de 1993.

À compter de 2009, chaque assuré sera soumis à des conditions de liquidation propre à sa génération mais, jusqu'à cette date, les modalités de calcul de la pension varient selon son année de naissance et l'année de sa demande de pension:

- Le nombre de salaires pris en compte dans le calcul du Sam augmente d'une unité par génération jusqu'à atteindre 25 pour les assurés nés en 1948, paramètre qui s'applique alors en 2008 à tous les assurés quelle que soit leur année de naissance ;

- La durée rémunérée par le Régime général passe de 150 trimestres pour les assurés nés avant 1944 à 160 trimestres pour tous en 2008 avant de croître à nouveau par génération à raison d'un trimestre par an ;

- La durée totale requise pour obtenir le taux plein est de 160 trimestres pour tous les assurés prenant leur retraite entre 2003 et 2008, puis elle augmente par génération au même rythme que la durée rémunérée par le Régime général. En prenant appui sur les projections de l'Insee, le Conseil d'Orientation des Retraites estime que la durée requise à partir de 2020 pourrait être de 167 trimestres $(41,75 \mathrm{ans})^{1}$.

Comme contrepartie de ce durcissement global des paramètres de calcul de la pension, plusieurs mesures visent plus particulièrement certains assurés.

- Pour les assurés faiblement rémunérés durant leur carrière et liquidant au taux plein, les règles de calcul du minimum contributif sont modifiées. D'une part, le montant est majoré de $3 \%$ en 2004, 2006 et 2008 mais, à compter du $1^{\mathrm{er}}$ juillet 2005, la majoration ne porte que sur les périodes cotisées par l'assuré. D'autre part, le minimum est servi au prorata de la durée effectuée au Régime général pour les polypensionnés dont la durée validée tous régimes dépasse la durée d'obtention du taux plein.

- Pour les assurés liquidant à taux réduit, la décote par trimestre manquant est diminuée de moitié entre les générations 1943 et 1953 et le taux minimal de liquidation passe ainsi de $25 \%$ à 37,5\%.

- Une surcote est introduite pour les assurés partant en retraite au-delà de 60 ans avec une durée d'assurance supérieure à celle requise pour prétendre au taux plein ; la pension, avant comparaison au minimum, est majorée de $0,75 \%$ par trimestre cotisé excédentaire, quel que soit le régime.

- Les salaires ne validant pas de trimestre sont neutralisés dans le calcul du Sam et, pour les assurés relevant d'un régime aligné, le calcul est effectué au prorata de leur durée d'assurance dans chacun des régimes.

- Les assurés ayant des carrières longues (conditions de durée totale d'assurance, de durée cotisée et d'âge de début d'activité) ont la possibilité de partir de façon anticipée, dès 56 ans.

Par ailleurs, la réforme réaffirme le système d'indexation appliqué depuis 1987 : l'évolution des prix hors tabac détermine les coefficients d'actualisation des salaires portés aux comptes, la revalorisation des pensions et l'augmentation conventionnelle du minimum contributif. Le salaire plafond reste quant à lui indexé sur l'évolution des salaires.

La réforme ne revient pas sur les retraites versées aux assurés ayant fait valoir leurs droits avant l'entrée en vigueur des mesures. Seule la pension des nouveaux retraités est donc potentiellement affectée par la modification des conditions de liquidation. Les pensions étant indexées sur les prix, le pouvoir d'achat au départ en retraite est donc maintenu sur toute la durée de service de la pension. 


\section{Les outils de projection retraite}

Les outils de projection utilisés en matière de retraite sont des outils d'exploration prospective. Ils permettent de déceler et d'anticiper, sous des contextes socio-économiques divers, des défaillances et des dérives du système et d'examiner une variété de stratégies correctrices. À cet égard, les choix méthodologiques qui président à leur construction sont étroitement liés aux préoccupations politiques (Vernière, 2001 : 6-7). À la modélisation macro-financière en équilibre général statique, centrée sur les interactions du système de retraite avec l'économie, ont ainsi succédé les approches générationnelles (comptabilité générationnelle, modèles à générations imbriquées) puis individuelles, pour répondre à la nécessité de mesurer les répercussions des déséquilibres financiers imminents sur la répartition « verticale » et « horizontale » des efforts à consentir.

Ces modélisations abordent des problématiques différentes et les plus récentes sont venues suppléer les outils préexistants, mais sans les remplacer. En se limitant aux évaluations reprises dans les rapports du Conseil d'orientation des retraites, trois méthodes coexistent ainsi au sein des administrations et des régimes: les évaluations sur cas types, les modèles générationnels et la microsimulation ${ }^{2}$.

\section{Les cas types}

L'évaluation à partir de situations individuelles théoriques (carrière au Smic, au plafond ...) est largement utilisée, car, par nature, elle dispense d'un lourd travail de collecte de données. Elle convient donc particulièrement pour les exercices de comparaisons internationales (Chagny et al., 2001). Toutefois, comme le souligne Daniel (1999), des précautions méthodologiques doivent être prises et il convient notamment de définir le cas type en fonction des différents contextes institutionnels étudiés ; par exemple, ne pas se limiter aux caractéristiques du bénéficiaire lorsque les prestations sont liées à la configuration familiale. Aussi, plutôt que de faire référence à des situations individuelles, les cas types doivent-ils être définis plus généralement comme des «unités de population parfaitement spécifiées en regard des 'critères discriminants' pour les opérations étudiées » Lagarde (1985: 275).

L'évaluation sur cas types relève davantage d'une volonté de " comprendre les logiques à l'œuvre " que de porter un diagnostic synthétique sur le système (Daniel, op.cit. : 25). Dans l'analyse des systèmes de retraite, l'approche est classiquement utilisée pour mesurer les conséquences des modifications de la législation vieillesse sur le taux de remplacement du dernier salaire ou du salaire moyen par la pension.

Le modèle Oscarie de la Drees, développé initialement par Dupuis et El Moudden (2000), permet par exemple de calculer les prestations versées par la Cnav, l'Arrco et l'Agirc pour un individu type défini par différentes caractéristiques : la génération, le sexe, le nombre d'enfants, l'âge d'entrée dans la vie active, le statut (cadre - non cadre), le profil de salaire, l'âge de liquidation et l'âge du décès (Raynaud, 2002). Oscarie a produit plusieurs chiffrages, rapportés entre autres par le Cor (2002, 2004 : 61-90) et la CCSS (Commission des Comptes de la Sécurité Sociale, 2003 : Ch. 10-4), qui éclairent sur les propriétés redistributives du système de retraite et les incidences de la réforme de 2003.

L'absence d'indication sur la représentativité des cas types utilisés représente le principal inconvénient de la méthode, car elle empêche toute évaluation à un niveau plus global, celui du régime notamment. L'utilisation de cas types pour des projections financières supposerait de déterminer au préalable des situations individuelles réellement représentatives et dont la représentativité dans la population pourrait être mesurée. Les améliorations récentes de la méthode vont dans ce sens : pour mesurer l'impact individuel de la réforme des retraites de 2003, Raynaud (2004) recourt aux cas types représentatifs des assurés aux carrières continues de la génération 1948, identifiés par une procédure statistique développée par Koubi (2005). Cependant, à défaut de disposer d'une approche similaire pour déterminer les représentants d'assurés aux carrières discontinues, Raynaud introduit arbitrairement des «accidents" à ces trajectoires, condamnant de fait le passage à une approche globale. 


\section{La macro-projection}

La macro-projection relève d'une approche agrégée. La population totale est ventilée en classes supposées homogènes (génération $\times$ sexe $\times$ catégorie sociale ou âge de fin d'études, par exemple) et la projection porte sur les individus présentant les caractéristiques économiques et démographiques moyennes de leur classe.

La maquette Mars du Ministère des finances pour les régimes de retraite du secteur privé Cnav, Arrco et Agirc (Bellone et Vincent, 2004), le modèle «global » de la Cnav (Tanchoux, 1989), ou encore le modèle Ariane pour la fonction publique d'État, appartiennent à cette catégorie.

Ces modèles ont pour atout leur simplicité d'appréhension et d'usage. En contrepartie, ils ne rendent pas compte de la dispersion au sein des classes et d'une éventuelle évolution de cette dispersion (biais d'agrégation). Aussi, les projections de pension ou d'âge de départ en retraite ne restent-elles pertinentes que si les disparités entre les trajectoires individuelles sont limitées. En revanche, en cas de non-linéarité de la formule de calcul de la pension, la multiplicité des parcours entraîne mécaniquement une inflation des erreurs d'estimation (Bac et al., 2003).

\section{La microsimulation}

La microsimulation repose sur « une description et/ou une modélisation des comportements au niveau des unités microéconomiques » (Legendre, 2004 : 19) et se fonde sur un échantillon jugé représentatif du système à étudier. Aujourd'hui considérés comme incontournables pour aborder les questions de disparités inter-individuelles et de redistribution, les modèles de microsimulation font l'objet d'une abondante littérature et plusieurs écrits récents dressent une synthèse sur les principes de la méthode, ses déclinaisons et ses applications (Chambaz et Le Minez, 2003 ; Dupont et al., 2004 ; Legendre, op.cit. ; Privat, 2005).

Les modèles de microsimulation cherchent à rendre compte de l'hétérogénéité des situations individuelles et, dans l'analyse du système de retraite français, leur champ d'application s'est naturellement orienté en priorité vers le Régime général.

L'Insee a développé le modèle Destinie (Insee, 1999 ; Bonnet et Mahieu, 2000b ; Bardaji et al., 2003) et l'a récemment prolongé par un module dédié au secteur public (Bardaji et al., 2004). L'outil a été largement utilisé pour la préparation et le suivi des réformes (Bardaji et al., 2002 ; Cor, 2002, 2004 ; CCSS, 2003) et a amélioré la connaissance du système de retraite, notamment sur ses effets redistributifs (Bonnet et Mahieu, 2000a; Dantec et al., 2000; Walraët et Vincent, 2003) et ses incidences sur les comportements de départ en retraite (Pelé et Ralle, 1999; Mahieu et Sédillot, 2000 ; Buffeteau et Godefroy, 2005).

La Cnav s'est dotée en 2004 de son propre modèle de microsimulation. Prisme reprend les bases du modèle Artémis (Debrand et al., 2004 ; Privat, op.cit.), mais retient un pas trimestriel et non plus annuel et s'enrichit, entre autres, d'un module de comportement de départ en retraite. Ses premiers chiffrages ont été rendus publics lors de la CCSS de septembre 2005.

Les modèles de projection de retraites sont dynamiques. Ils simulent, individu par individu, les évènements démographiques (natalité, décès) et professionnels (salaires, passages par le chômage, inactivité ...) à partir de fonctions d'évolution et de comportements estimées préalablement. Le modèle de microsimulation s'apparente alors à « un générateur de trajectoires individuelles » (Dupont et al., op.cit. : 176). L'approche est pragmatique : elle s'effectue au niveau de la prise de décision (O’Donoghue, 2001) et permet de réaliser des « expériences réelles » (Legendre, op.cit. : 20, 25). 
Plusieurs critiques ont néanmoins été soulevées à l'égard de la microsimulation. Des différentes synthèses réalisées sur ses limites ( $c f$. entre autres Van Imhoff et Post, 1997 ; O'Donoghue, op.cit. ; Legendre, op.cit.), sept points peuvent être relevés.

- Une incertitude sur le pouvoir prédictif du modèle

La projection par microsimulation présente plusieurs sources d'imprécision (Caldwell, 1996; Van Imhoff et Post, op.cit.; Dupont et al., op.cit.) : erreurs d'échantillonnage portant sur les individus sur lesquels portent la simulation et sur lesquels sont fondées les estimations, erreur de spécification des relations économétriques (linéaire vs. non linéaire, omission de variables ...), mauvaise qualité de données ou des paramètres utilisés, etc. Finalement, « les marges réelles de précision des estimations $[\ldots]$ ne peuvent pas vraiment être fournies » (Dupont et al., op.cit. : 178).

- Un déséquilibre entre la sophistication du modèle et le niveau de désagrégation pertinent des résultats

Le souci d'être au plus proche de la réalité fait la force des modèles de microsimulation, mais conduit aussi à multiplier les sources d'erreurs possibles (biais d'endogénéité, erreurs de spécification) sans que celles-ci puissent être toutes contrôlées. En particulier, le pouvoir prédictif du modèle décroît avec le nombre de variables explicatives («aléas de spécification», cf. Van Imhoff et Post, op.cit.). Le raffinement des hypothèses ne trouve donc pas son équivalent en termes de résultats, car les intervalles de confiance - qu'ils soient explicités ou non - contraignent à un niveau d'agrégation suffisant pour garantir la validité des résultats. L'homogénéité des classes retenues reste alors conditionnée aux critères de stratification (secteur d'activité, PCS, âge de fin d'études, par exemple).

- Des modèles « boites noires »

La microsimulation contourne l'impossibilité d'expliciter la complexité et la diversité des situations individuelles en recourant à un échantillon de ces situations pour mieux appréhender le fonctionnement de l'ensemble du système. Ce faisant, l'approche stochastique de la méthode et la multiplicité des paramètres introduits pour simuler les situations et comportements, compromettent toute résolution analytique et rendent complexe toute justification à un niveau "supraindividuel » quant aux différences entre variantes et/ou entre exercices.

\section{- Une approche en équilibre partiel}

En raison de la complexité que cela générerait, notamment pour limiter les " aléas de spécification », les interactions entre individus sont rarement modélisées dans les modèles de microsimulation (hormis au sein du ménage, $c f$. Walraët et Vincent, op.cit.). L'approche repose donc sur un équilibre partiel, sans que les comportements individuels modifient les grandeurs macro.

Plusieurs approches ont toutefois été récemment développées pour lier " micro » et « macro », voire assurer un bouclage macroéconomique. La solution la plus simple consiste à alimenter le modèle de microsimulation par un modèle macroéconomique en équilibre général. Les deux modèles fonctionnent indépendamment l'un de l'autre, séquentiellement (approche top down), sans qu'un lien formel ait à être établi (Robillard et al. 2001). D'autres approches tentent, à l'opposé, d'établir cette cohérence théorique pour rendre compte des effets de retour des comportements individuels sur les variables macro, mais elles restent rares, car relativement lourdes à mettre en œuvre (cf. e.g. Bourguignon et al., 2003).

\section{- Des comportements individuels figés}

En microsimulation, la modélisation consiste en un ensemble de relations économétriques dont les paramètres ont été estimés soit sur l'échantillon servant de base à la simulation, soit sur des populations ad hoc. Schématiquement, cela revient à appliquer à un individu les probabilités de transition entre deux états (emploi $\rightarrow$ chômage, par exemple), observées antérieurement sur la population présentant les mêmes caractéristiques (même sexe, même catégorie sociale ...). 
Cela soulève deux remarques : la première, sur la difficulté des modèles de microsimulation à intégrer la modification du comportement des agents, lorsque l'environnement dans lequel ils évoluent diffère de celui sur lequel les lois qui les régissent ont été estimées (critique de Lucas, 1976) ; la seconde, sur la confusion faite au sein de ces modèles entre paramètres et variables, variables exogènes et variables endogènes, de ce qui est relatif à l'individu et de ce qui dépend de l'environnement économique.

\section{- Une cohérence externe difficile à assurer}

Dans les modèles de microsimulation, la cohérence externe, c'est-à-dire la coïncidence entre les hypothèses macro (économiques et démographiques) avec celles résultant de l'agrégation des résultats, n'est pas assurée intrinsèquement et doit être vérifiée a posteriori. Or le calage des hypothèses et celui des prévisions sur les réalisations peuvent se révéler particulièrement délicats à effectuer lorsqu'ils supposent de procéder à de nouvelles estimations, de modifier la spécification de certaines lois et/ou d'altérer certaines des caractéristiques des individus. Cette critique trouve toute sa pertinence dans un contexte de révision réglementaire quasi-permanente où un modèle sera jugé sur sa capacité à «s'auto-corriger » pour intégrer les modifications de comportements.

- Une mécanique lourde, difficile à maintenir

Les modèles de microsimulation dynamiques font intervenir un grand nombre de microprocessus qui réclament de lourds investissements en collecte de données, modélisation et validation. Le lien étroit entre ces modèles et l'échantillon de données individuelles sur lesquels ils reposent, les attache au système qu'ils sont censés représenter (Van Imhoff et Post, op.cit. : 797) et contrarie bien souvent leur transposition à d'autres systèmes (autres régimes de retraite, par exemple). Par ailleurs, toute modification affectant les variables et/ou les équations est hasardeuse, car la multiplicité des relations économétriques soulève le risque d'une incohérence interne (variables sur-identifiées, notamment) et le gain net en pouvoir prédictif est difficile à apprécier. Le manque de flexibilité d'un modèle de microsimulation est donc une entrave majeure à sa maintenance et à sa pérennité.

La modification quasi-permanente de la législation vieillesse attendue à la suite de la réforme des retraites de 2003 requiert de disposer - peut-être plus que précédemment - d'outils d'évaluation adaptatifs pour lesquels la dimension individuelle est centrale. Si les approches agrégées échouent sur ce point et sont jugées trop simplificatrices au regard des transformations récentes du marché du travail, les méthodes de cas types et la microsimulation ne répondent que partiellement à l'objectif : les premières sont trop théoriques et inadaptées à l'exercice de projection financière, les secondes sont complexes, peu flexibles et, finalement, peu appropriées à la description d'un système en constante évolution. Une approche intermédiaire entre l' « agrégé » et l' « individuel » doit donc être recherchée. 


\section{Le modèle Eclipss}

Deux composantes interviennent dans l'évolution de la pension attribuée aux flux successifs de retraités : d'une part, un effet noria lié à la modification des carrières suivies d'une génération à l'autre, d'autre part, un effet réglementaire tenant aux changements affectant le calcul des pensions. Le modèle Eclipss vise précisément à prendre en compte ces deux aspects pour projeter la pension servie aux retraités du secteur privé. L'acronyme signifie «Évolution des Carrières, des conditions de LIquidation et de la Pension de la Sécurité Sociale » et fait d'ailleurs clairement apparaitre ces deux dimensions.

Le principe de modélisation reprend à chacune des approches présentées précédemment tout en essayant de répondre à leurs limites.

- D'une part, le modèle s'inscrit dans la lignée des modèles générationnels, mais il retient huit individus types et non un seul, ce qui limite la dispersion en termes d'éléments de droit à pension et réduit les écarts entre pension moyenne et moyenne des pensions liés aux non linéarités de la formule de calcul.

- D'autre part, les profils de carrières retenus sont issus de groupes de parcours professionnels réels. Identifiés par une analyse de classification tenant compte de leur caractère longitudinal (Briard, 2004), ce qui évite ainsi de recourir à des critères de stratification a priori et assure la pertinence et la représentativité de chaque profil de carrière. Un des atouts du modèle est donc de pouvoir évaluer de façon ciblée les impacts d'une modification des règles de calcul des pensions selon une méthode proche des cas types, mais en retraçant des trajectoires professionnelles réalistes, en levant l'hypothèse de stabilité de l'environnement et en disposant d'une indication sur la proportion d'individus concernés. L'approche peut ainsi être qualifiée de méthode de «carrières types dynamiques pondérées ».

\section{Des carrières types ...}

La spécificité du modèle réside dans la représentation qui est faite du marché du travail autour de huit profils de carrières qui peuvent être résumés de la façon suivante :

Groupe 1 : carrière salariale courte dans le secteur privé,

Groupe 2 : carrière effectuée en majorité hors du secteur privé,

Groupe 3 : carrière avec sortie précoce du marché du travail par invalidité,

Groupe 4 : carrière à salaires faibles avec inactivité entre 25 et 35-40 ans,

Groupe 5 : carrière précaire avec interruptions, à salaires moyens,

Groupe 6 : carrière continue à salaires moyens,

Groupe $7:$ carrière longue et continue à salaires élevés,

Groupe 8 : carrière continue avec salaires souvent au plafond.

\section{... dynamiques ...}

En projection, huit carrières types sont construites par génération. Elles retracent le déroulement de la carrière salariale et font mention de la durée d'assurance à 60 ans avec une décomposition selon l'origine des trimestres validés : chômage, maladie, invalidité, service militaire, activités effectuées hors du champ du Régime général ou encore avantages familiaux tels que la majoration de durée d'assurance pour enfants (MDA) et l'assurance vieillesse des parents au foyer (AVPF). Pour les générations 1935 à 1940, les carrières types s'apparentent à des carrières moyennes; pour les générations 1941 à 1960, seule les fins de vie active sont projetées; enfin, pour toutes les autres générations, l'intégralité des parcours professionnels est recréée, génération par génération, selon des hypothèses propres à leur groupe. 


\section{... pondérées}

La représentativité des profils de carrières est évaluée par analyse discriminante sur les comptes individuels de 500000 assurés du Régime général nés entre 1935 et 1960 et observés jusqu'à 39 ans puis posée comme hypothèse pour les générations suivantes. Son évolution témoigne d'un double mouvement : l'augmentation de l'activité féminine et la montée quasi continue du chômage. Elle fait apparaître un remplacement progressif des carrières courtes effectuées dans le secteur privé (groupes 1 et 4) par des carrières plus longues (groupes 5 et 6 ), mais pour lesquelles les interruptions pour cause de chômage ou d'inactivité occupent une place croissante en se substituant aux retraits volontaires du marché du travail.

\section{Des comportements de départ en retraite différenciés}

La spécification du comportement de départ en retraite reste un point délicat dans les modèles de projection de pensions. Aucune réponse vraiment satisfaisante n'a effectivement été proposée quant aux déterminants de la décision de l'âge de départ et à la prise en compte des contraintes posées par la demande de travail sur la possibilité de continuer de valider des trimestres d'assurance et de disposer de ressources au-delà de 60 ans, âge minimal pour faire valoir ses droits à une pension.

En distinguant différents déroulements de parcours professionnels, la modélisation par carrières types se démarque des spécifications classiques des comportements de départ en retraite. Dans le modèle Eclipss, les comportements d'activité au-delà de 60 ans sont les suivants :

Groupes 1 et 2 : départ en retraite à 60 ans pour une partie des assurés, inactivité jusqu'à 65 ans pour l'autre partie,

Groupe 3 : départ en retraite à 60 ans au taux plein au titre de l'invalidité,

Groupes 4, 6, 7 et 8 : activité salariée dans le secteur privé jusqu'à l'obtention du taux plein,

Groupe 5 : chômage indemnisé jusqu'à l'obtention du taux plein.

Avant la réforme de 2003, l'hypothèse très largement retenue du départ dès l'obtention du taux plein se justifiait par les fortes pénalités financières appliquées sur la pension en cas de liquidation précoce et par la désincitation à poursuivre une activité une fois les conditions remplies, les trimestres excédentaires n'étant pas valorisés (Blanchet et Pelé, 1997 ; Pelé et Ralle, 1999). En diminuant la décote et en instaurant une surcote, la dernière réforme vise à introduire plus de flexibilité dans la décision de partir en retraite et remet donc en question cet argument.

En 2003, 7,9\% des droits contributifs directs étaient attribués à taux réduit. Compte tenu des comportements d'activité au-delà de 60 ans spécifiés dans le modèle, ces départs se concentreraient dans les groupes 1 et 2 où les assurés sont sortis du champ du Régime général au moment de leur départ en retraite. Bien que cela conduise vraisemblablement à une sous-estimation, cette proportion peut être posée comme constante en projection à défaut de disposer d'information sur son évolution. Sous l'hypothèse que la durée requise pour le taux plein plafonne à 167 trimestres à l'horizon 2050, grâce à leur longue durée d'activité totale, les assurés du groupe 2 ne subiraient aucune décote de leur taux de liquidation en partant en retraite dès 60 ans ; $27 \%$ des assurés du groupe 1 liquideraient alors leurs droits à taux réduit en 2003 et deux tiers en 2050. 


\section{Une application : l'évolution de la pension suite à la réforme de 2003}

La réforme des retraites de 2003 est venue modifier le calcul des pensions à deux niveaux. D'une part, elle augmente la durée d'assurance pour obtenir une pension pleine et entière et en fixe les règles d'évolution sur le long terme. D'autre part, elle limite les conséquences de ce durcissement des paramètres de liquidation pour des catégories particulières d'assurés (salariés ayant cotisé à plusieurs régimes ou faiblement rémunérés, notamment) en modifiant les modalités de calcul de leur pension.

Ces mesures amènent à s'interroger sur l'évolution des disparités entre retraités au fil des générations que celles-ci soient dues aux différences de parcours professionnels ou de modalités de départ en retraite. Pour appréhender les mécanismes mis en œuvre et évaluer leurs conséquences sur les populations visées, le cadre de projection retenu est le suivant :

- La représentativité des huit groupes de carrières est supposée rester conforme à celle de la génération 1960 en raison du ralentissement attendu des taux d'activité des femmes nées à partir de la fin des années soixante (Nauze-Fichet et al., 2003 ; Afsa et Buffeteau, 2005) et de la stabilisation des effets des tensions sur le marché du travail, ne serait-ce qu'à court terme (OFCE, 2004).

- À compter de 2005, les salaires évoluent selon deux hypothèses, dans un premier temps comme les prix, dans un second temps à un rythme de $2 \%$ à $1,8 \%$ par an. Dans les deux cas, le taux de chômage se stabilise à $8 \%$ à partir de 2010 .

- Les modalités de calcul des pensions sont celles fixées par la réforme à deux exceptions : la mesure relative aux départs anticipés n'est pas prise en compte et la majoration du minimum contributif en fonction de la durée cotisée est appliquée à compter de 2006 et non du $1^{\text {er }}$ juillet 2005.

- Enfin, on suppose que les salaires perçus au-delà de 60 ans n'entrent pas dans le calcul du Sam.

Afin de saisir les seules conséquences réglementaires de la réforme, une hypothèse de reproduction à l'identique des carrières est nécessaire. Dans un premier temps, les carrières types de la génération 1940 (60 ans en 2000) sont donc retenues, seuls les salaires sont susceptibles d'évoluer. Dans un second temps, l'approche normative est dépassée en tenant compte de la dynamique de déformation des parcours professionnels d'une génération à l'autre (périodes de chômage plus ou moins nombreuses par exemple).

\section{L'effet réglementaire}

Sous l'hypothèse que les assurés font valoir leurs droits lorsqu'ils accèdent au taux plein, la modification des paramètres de calcul a un impact limité sur le montant des pensions, le recul de l'âge de départ en retraite compensant en partie les effets de l'allongement de la durée requise. Aussi, sous les seules modifications programmées en 1993, l'augmentation globale du montant des pensions aurait-elle été de $45 \%$ par rapport à 2000 , soit une croissance de 5 points supérieure à celle résultant d'un scénario « avec réforme de $2003 »$ ( $c f$. tableau 1).

Sur la période 2000-2008, les modifications réglementaires introduites en 2003 bénéficient aux assurés aux revenus les plus faibles. Par rapport au seul cadre de la réforme de 1993, grâce à la majoration du minimum contributif dont ils bénéficient en cas de départ au taux plein, et à la baisse de la décote lorsqu'ils liquident leurs droits à taux réduit, les assurés des groupes 1 et 4 ont une pension qui croît de façon plus soutenue : respectivement au rythme de $+0,18 \%$ et de $+0,43 \%$ par an en moyenne avec réforme contre $-0,13 \%$ et $0,37 \%$ sans réforme ( $c f$. tableau 1 ). 
Les assurés les plus lésés par la réforme de 2003 sont ceux qui ont suivi les carrières les plus longues dans le secteur privé. En dépit du recul de leur âge de départ en retraite pour obtenir le taux plein, leur pension augmente moins que dans un scénario «sans réforme ». Les écarts restent cependant limités : au plus 5 points en 2020 et au plus 7 en 2050. Certains d'entre eux, polypensionnés, peuvent par ailleurs voir leur situation s'améliorer : dans les groupes 2 et 3 , la pension est portée au minimum contributif en début de projection et les assurés sortent du dispositif grâce à la réduction du nombre de salaires entrant dans le calcul de leur salaire de référence et bénéficient alors de pensions plus élevées.

Tableau 1.

Évolution de la pension à la liquidation

avec ou sans réforme de 2003

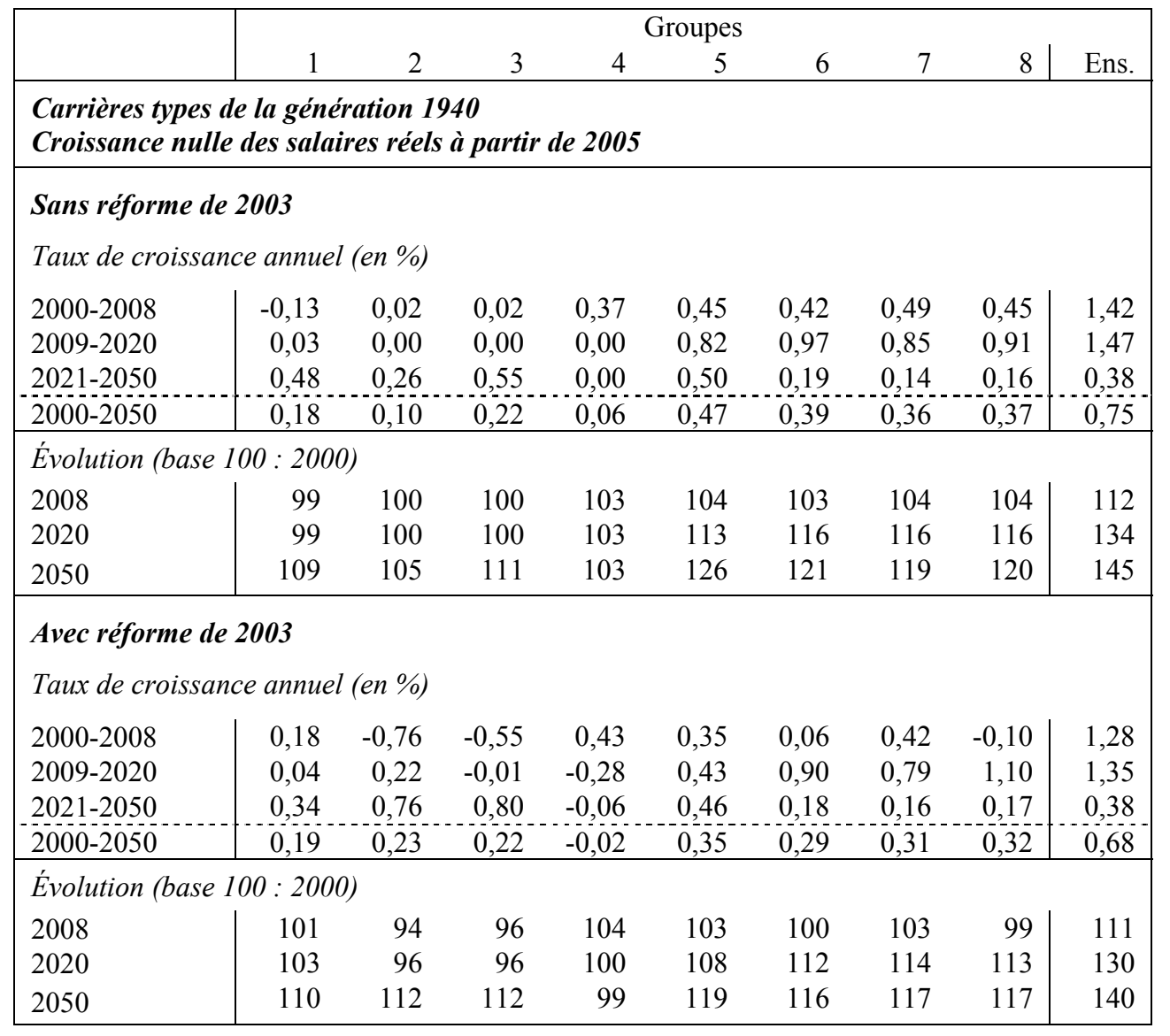

Le rythme de renouvellement des générations peut être saisi en levant l'hypothèse de stabilité de l'environnement, ne serait-ce que partiellement. On peut par exemple supposer que les profils salariaux se modifient d'une génération à l'autre sans que le déroulement des parcours professionnels en soit affecté ni, par conséquent, les durées d'assurance validées au terme de la vie active.

En s'appuyant sur les prévisions réalisées par l'OFCE en octobre 2004 (OFCE, 2004), on envisage désormais une hypothèse haute de croissance des salaires réels selon laquelle ils augmentent de $2 \%$ par an jusqu'en 2009 , de $1,9 \%$ entre 2010 et 2015 puis de $1,8 \%$.

À moyen terme, les carrières des futurs retraités sont en grande partie connues, seules les dernières années de vie active des assurés aux parcours les plus longs sont sensibles aux conditions du marché du travail et l'hypothèse de croissance des salaires n'affecte l'évolution de la pension qu'à long terme, au-delà de 2020. Ainsi, suite à la réforme de 2003, la pension servie à la liquidation croît globalement de $30 \%$ à $33 \%$ entre 2000 et 2020 ( $c f$. tableaux 1 et 2) et les conséquences de l'augmentation plus soutenue des rémunérations apparaissent sur la période 2020-2050 avec un écart de plus de 2 points du rythme de croissance annuel des pensions : $0,38 \%$ dans le cas où les salaires suivent les prix, 2,43\% s'ils croissent à un rythme proche de $2 \%$ par an. 
Les rémunérations perçues durant la vie active n'interviennent pas dans le montant versé à la retraite dès lors que la pension est portée au minimum contributif. Dans ce cas, les disparités entre les générations successives restent limitées ; le montant servi est revalorisé chaque année comme les prix et seules pourraient alors jouer les différences en termes de durée d'assurance rémunérée par le Régime général (proratisation) et de proportion des périodes cotisées dans cette durée. Toutefois, dans l'hypothèse d'une reproduction à l'identique des parcours professionnels d'une génération à l'autre (les carrières de la génération 1940 dans le cas présent), les disparités entre générations ne peuvent alors tenir qu'aux modifications paramétriques du calcul des pensions interviennent.

Les assurés du groupe 4, faiblement rémunérés durant leur carrière, ont leur pension portée au minimum sur presque tout l'horizon de projection et son évolution est donc quasi nulle jusqu'en 2050 ; à l'inverse, l'évolution de la pension des assurés aux salaires les plus élevés (groupes 5 à 8 ) est en lien étroit avec la progression des salaires et une augmentation en termes réels de l'ordre de $2 \%$ par an se traduit sur la pension par plus de 2 points de croissance supplémentaires chaque année entre 2021 et 2050 (de 2,32\% à 2,62 \% contre au plus $0,46 \%$ dans l'hypothèse d'une croissance nulle des salaires).

Tableau 2.

Évolution de la pension à la liquidation

effet de la croissance des salaires

\begin{tabular}{|c|c|c|c|c|c|c|c|c|c|}
\hline & \multicolumn{9}{|c|}{ Groupes } \\
\hline & 1 & 2 & 3 & 4 & 5 & 6 & 7 & 8 & Ens. \\
\hline \multicolumn{10}{|c|}{$\begin{array}{l}\text { Carrières types de la génération } 1940 \\
\text { Croissance des salaires réels de } 2 \% \text { à } 1,8 \% \text { par an } \\
\text { Avec réforme de } 2003\end{array}$} \\
\hline \multicolumn{10}{|c|}{ Taux de croissance annuel (en \%) } \\
\hline $2000-2008$ & 0,18 & $-0,76$ & $-0,55$ & 0,43 & 0,35 & 0,06 & 0,42 & $-0,10$ & 1,28 \\
\hline $2009-2020$ & 0,04 & 0,22 & $-0,01$ & $-0,28$ & 0,43 & 1,20 & 1,23 & 1,39 & 1,58 \\
\hline $2021-2050$ & 0,91 & 1,62 & 1,64 & $-0,03$ & 2,32 & 2,59 & 2,62 & 2,55 & 2,43 \\
\hline $2000-2050$ & 0,41 & 0,57 & 0,54 & 0,00 & 1,07 & 1,30 & 1,38 & 1,32 & 1,54 \\
\hline \multicolumn{10}{|c|}{ Évolution (base $100: 2000$ ) } \\
\hline 2008 & 101 & 94 & 96 & 104 & 103 & 100 & 103 & 99 & 111 \\
\hline 2020 & 103 & 96 & 96 & 100 & 108 & 115 & 119 & 116 & 133 \\
\hline 2050 & 123 & 133 & 131 & 100 & 170 & 191 & 198 & 192 & 214 \\
\hline
\end{tabular}

\section{L'effet de la déformation des parcours professionnels}

Si la prise en compte de la croissance des salaires au fil des générations permet d'apprécier le rythme de renouvellement des générations, elle ne fournit qu'une mesure partielle de l'effet noria. Pour dépasser complètement le cadre normatif, l'hypothèse d'une rémanence des carrières d'une génération sur l'autre doit être levée; les carrières types des différentes générations en âge de partir en retraite à l'horizon 2050 - et non plus seulement celles de la génération 1940 - doivent donc être désormais retenues.

Dans ce contexte, on estime que l'âge moyen de départ augmente de près d'un an entre 2000 et 2050 passant de 62,3 à 62,9 ans ${ }^{3}$. Cette évolution s'effectue en deux phases. Dans un premier temps, l'âge moyen de départ baisse sous l'effet de l'allongement de la durée d'assurance validée par les générations du baby boom plus actives et qui bénéficient plus largement de l'assurance vieillesse des parents au foyer (AVPF), instaurée en 1972. À partir de 2012, le durcissement des paramètres de liquidation conduit à un recul de l'âge de départ en retraite atteignant jusqu'à plus d'un an et demi à l'horizon 2050 ; pour les assurés nés à partir de 1950, les durées d'assurance validées ne sont alors plus suffisantes pour satisfaire aux conditions. Les derniers concernés sont naturellement les assurés des groupes 6,7 et 8 aux carrières les plus longues. Ils accèdent au taux plein dès 60 ans en début de projection, commencent à différer leur départ en 2015 puis partent respectivement à 62,63 et 64 ans en 2050. 
Ce recul de l'âge de départ atténue les conséquences financières de la modification des paramètres de calcul. Sans cet ajustement, sous l'hypothèse d'une croissance des salaires réels proche de $2 \%$ par an, la pension n'augmenterait que de $71 \%$ entre 2000 et 2050 soit un écart de plus de 26 points avec la situation de référence ( $c f$. tableau 3). Les assurés aux carrières les plus longues (groupes 5 à 8 ) seraient alors particulièrement affectés avec un écart de 18 à 46 points, le montant servi à la liquidation en 2050 augmentant au plus de $56 \%$ par rapport à 2000 .

Outre l'allongement de la durée d'assurance, la réforme de 2003 a modifié les modalités de calcul des pensions pour certaines catégories d'assurés.

La baisse de la décote consiste ainsi à appliquer des pénalités financières moindres aux assurés qui font valoir leurs droits à taux réduit. Ainsi, pour les assurés du groupe 1 qui partent à 60 ans, le taux de liquidation passe de $25 \%$ à 37,5\% entre 2003 et 2013 . Cependant, la proportion d'assurés liquidant à taux plein dans le groupe étant supposée décroître de génération en génération ${ }^{4}$, la pension servie en moyenne dans le groupe baisse de $7 \%$.

Selon nos estimations, le minimum contributif concerne jusqu'à $45 \%$ des nouveaux retraités entre 2000 et 2050. Il s'agit des assurés du groupe 1 qui attendent 65 ans pour liquider leurs droits et ceux des groupes 2, 3, 4 et 5 qui partent en retraite avant 2020, 2026, 2048 et 2019 respectivement, éventuellement de façon discontinue. La proportion d'assurés bénéficiaires du minimum contributif est donc très variable selon les années en raison des effets de seuil inhérents à l'approche par individus représentatifs. Sa baisse doit par ailleurs être reliée aux hypothèses sur la représentativité des groupes, notamment le poids des carrières les moins bien rémunérées, décroissant jusqu'à la génération 1960 puis supposé constant en projection.

Les assurés qui font valoir leurs droits entre 2004 et 2008 appartiennent à des générations plus actives, l'allongement de la durée d'assurance validée permet une amélioration du coefficient de proratisation de la pension qui vient s'ajouter à la majoration de $3 \%$ du minimum contributif. Dans le groupe 4, cela se traduit en 2008 par une augmentation de $10 \%$ de la pension par rapport au montant moyen attribué en 2000. Le basculement dans les nouvelles règles de calcul applicables aux polypensionnés contrebalance cependant ces effets dans le groupe 3 où la pension baisse de $2 \%$. Les assurés du groupe 2 bénéficient quant à eux d'une hausse de leur salaire de référence, désormais calculé sur la seule base des années effectuées au Régime général; ils sortent ainsi du dispositif du minimum contributif jusqu'en 2013, après que leur pension a crû de $4 \%$ entre 2000 et 2008.

Enfin, en restant en activité au-delà de 60 ans, les assurés des groupes 4, 6, 7 et 8 peuvent bénéficier de la surcote bien que pour les assurés du groupe 4, dont la pension est portée au minimum, son montant soit nul. Compte tenu de l'hypothèse de départ en retraite dès le taux plein avec un recul de l'âge de la liquidation par année entière, le nombre maximal de trimestres sur lequel peut porter la surcote est cependant ici limité à trois et les montants calculés ne sont donc pas significatifs.

Depuis 1987, les pensions sont indexées sur les prix. Il en résulte que le montant de pension attribué à la liquidation est en termes réels celui qui sera théoriquement versé durant toute la retraite. Pourtant, même si à prix constant les pensions augmentent d'une génération à l'autre, dans le cas où les salaires augmentent plus vite que les prix, le pouvoir d'achat des retraités se dégrade relativement à celui des actifs. Les disparités entre générations n'apparaissent donc pas seulement entre les différentes cohortes de retraités au cours du temps, mais également entre classes d'âges à une date donnée.

Rapportée à l'évolution générale des salaires, de $2 \%$ à $1,8 \%$ par an, la pension moyenne baisserait ainsi de $17 \%$ entre 2000 et 2050 suite à la réforme de 2003 avec des pertes de pouvoir d'achat plus importantes pour les retraités aux pensions les plus faibles : $-56 \%$ dans le groupe 1 contre $-20 \%$ dans le groupe 7 par exemple ( $c f$. tableau 4). 
Tableau 3.

Évolution de la pension à la liquidation

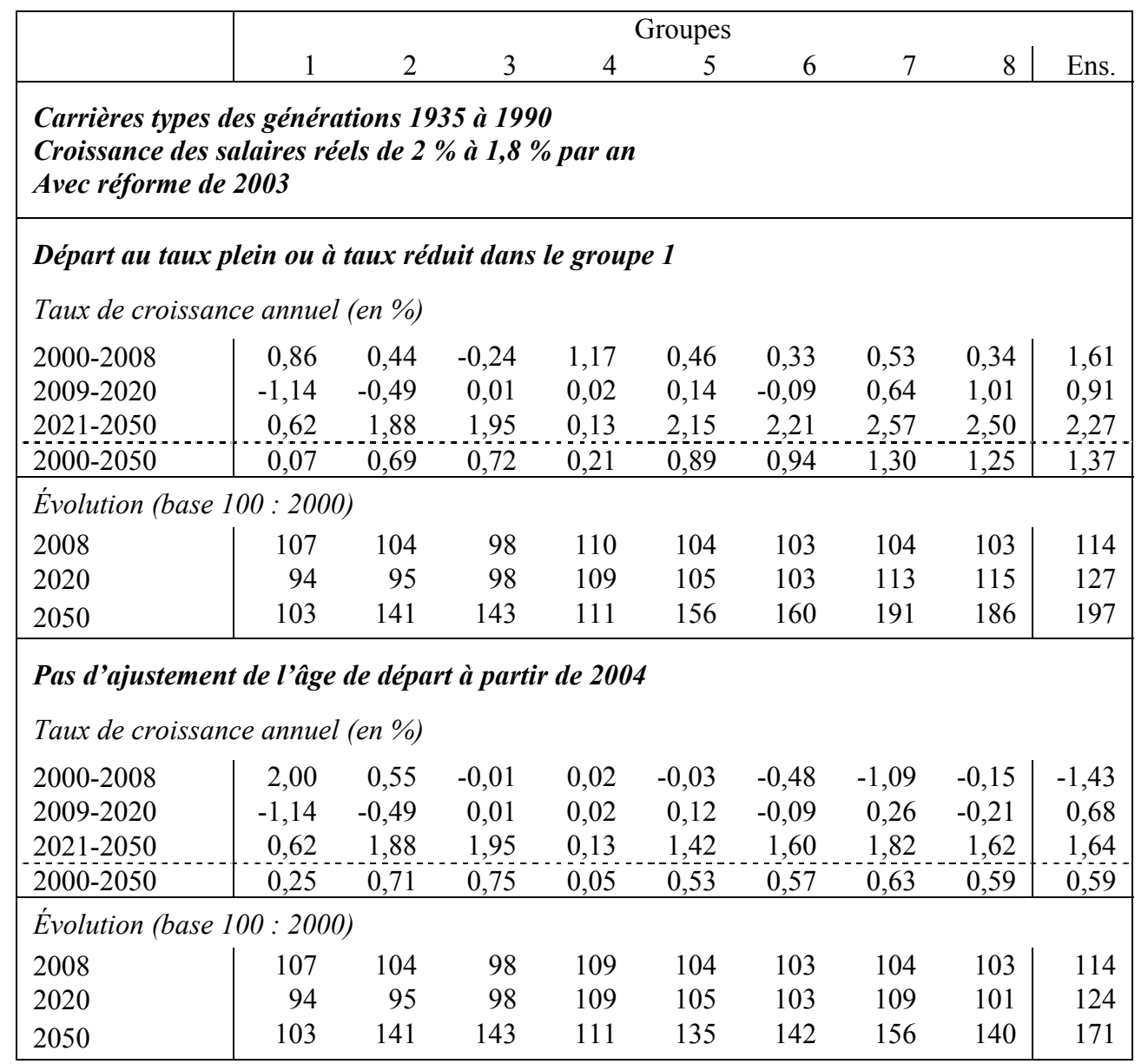

Tableau 4.

Évolution du ratio Pension / SMPT *

\begin{tabular}{|c|c|c|c|c|c|c|c|c|c|}
\hline & \multicolumn{9}{|c|}{ Groupes } \\
\hline & 1 & 2 & 3 & 4 & 5 & 6 & 7 & 8 & Ens. \\
\hline \multicolumn{10}{|c|}{$\begin{array}{l}\text { Carrières types des générations } 1935 \text { à } 1990 \\
\text { Croissance des salaires réels de } 2 \% \text { à } 1,8 \% \text { par an } \\
\text { Avec réforme de } 2003\end{array}$} \\
\hline \multicolumn{10}{|c|}{ Départ au taux plein ou à taux réduit dans le groupe 1} \\
\hline \multicolumn{10}{|c|}{ Taux de croissance annuel (en \%) } \\
\hline $2000-2008$ & 0,33 & 0,62 & 0,26 & 0,42 & 0,25 & 0,09 & 0,12 & 0,17 & 0,11 \\
\hline $2009-2020$ & $-2,94$ & $-2,30$ & $-1,81$ & $-1,80$ & $-1,68$ & $-1,91$ & $-1,19$ & $-0,83$ & $-0,92$ \\
\hline 2021-2050 & $-2,05$ & $-0,80$ & $-0,74$ & $-2,53$ & $-0,54$ & $-0,48$ & $-0,12$ & $-0,20$ & $-0,42$ \\
\hline $2000-2050$ & $-1,52$ & $-0,79$ & $-0,72$ & $-1,41$ & $-0,66$ & $-0,62$ & $-0,29$ & $-0,30$ & $-0,39$ \\
\hline \multicolumn{10}{|c|}{ Évolution (base $100: 2000$ ) } \\
\hline 2008 & 96 & 93 & 88 & 99 & 93 & 92 & 94 & 92 & 102 \\
\hline 2020 & 68 & 69 & 71 & 78 & 76 & 74 & 82 & 83 & 92 \\
\hline 2050 & 44 & 60 & 60 & 47 & 66 & 67 & 80 & 79 & 83 \\
\hline
\end{tabular}

* SMPT : Salaire Moyen Par Tête 


\section{Conclusion}

Dans la réflexion qui entoure de façon permanente l'évolution du système de retraite, le législateur doit s'interroger sur la nature des disparités, sur leurs causes, sur l'opportunité de les maintenir ou de les atténuer, sur les moyens à mettre en œuvre pour y parvenir et sur les conséquences que pourraient avoir ces actions. Actuellement, l'arrivée à l'âge de la retraite de générations nombreuses, entrées sur le marché du travail dans des conditions plus favorables que celles chargées d'assurer le financement de leur retraite, a rendu plus aiguë la problématique de la répartition des contributions et des prestations. La multiplicité des mesures prises en 2003 en est une des manifestations. La réforme témoigne d'une volonté de concilier solidarité entre les générations et justice sociale tout en préservant l'équité individuelle, autrement dit le maintien d'un lien fort entre parcours professionnel et retraite, entre effort contributif et pension.

Dans un tel contexte, la projection effectuée à partir de carrières types dynamiques et pondérées se révèle riche d'enseignements. Elle concilie la précision et la flexibilité requises pour évaluer et analyser l'évolution des pensions individuelles. Si l'approche reste agrégée, elle rend néanmoins compte des disparités de situation et de traitement, la modification des rapports au sein des générations et entre les générations, et permet d'appréhender les objectifs et les mécanismes alors mis en place.

Le durcissement des conditions de liquidation introduit par la réforme de 2003 conduit à un recul de l'âge de départ en retraite qui permet de limiter la baisse des pensions attribuées aux assurés ayant eu la possibilité de se maintenir sur le marché du travail. En revanche, les mesures visant à garantir un niveau minimal de retraite ne font que retarder les conséquences financières négatives et apparaissent insuffisantes sur le long terme. Du fait de la revalorisation des pensions sur les prix, l'allongement de la durée de la retraite conduit à creuser les disparités entre actifs et retraités. Aussi, après avoir justifié l'augmentation de la durée d'assurance pour obtenir une pension pleine et entière, l'augmentation de l'espérance de vie pourrait-elle légitimer une réforme des clauses d'indexation afin d'assurer une plus grande équité intergénérationnelle. 


\section{Bibliographie}

Afsa C. et Buffeteau S. (2005). «L'évolution de l'activité féminine en France : une approche par pseudo-panel », Insee, Document de travail, n ${ }^{\circ} \mathrm{G} 2005 / 02,33$ p.

Bac C., Bonnet C. et Raynaud E. (2003). «L'évolution de la pension moyenne dans les modèles de simulation à long terme des dépenses de retraite », Drees, Document de travail, n 33, 68 p.

Bardaji J., Sédillot B. et Walraët E. (2002). «Évaluation de trois réformes du Régime Général d'assurance vieillesse à l'aide du modèle de microsimulation Destinie », Insee, Document de travail, ${ }^{\circ} \mathrm{G} 2002 / 07,39 \mathrm{p}$.

Bardaji J., Sédillot B. et Walraët E. (2003). «Un outil de prospective des retraites : le modèle de microsimulation Destinie », Économie et Prévision, n 160-161, pp. 193-213.

Bardaji J., Sédillot B. et Walraët E. (2004). «Les retraites du secteur public : projections à l'horizon 2040 à l'aide du modèle de microsimulation Destinie», Insee, Document de travail, $\mathrm{n}^{\circ} 2004 / 14,31 \mathrm{p}$.

Bardaji J. et Walraët E. (2003). «Les retraites du secteur privé : évaluation du cœur de la réforme Fillon à l'aide du modèle de microsimulation Destinie », Insee, Note, nº 19-03/G210.

Bellone B. et Vincent A. (2004). «Présentation de la maquette retraites Mars - 2003 », Document de travail, $31 \mathrm{p}$.

Blanchet D. et Pelé L.-P. (1997). "Social Security and Retirement in France", NBER, Document de travail, $\mathrm{n}^{\circ} 6214,33 \mathrm{p}$.

Bonnet C. et Mahieu C. (2000a). «Taux de rendement interne par générations des régimes obligatoires de retraite : calcul à l'aide d'un modèle de microsimulation dynamique », Revue Économique, vol. 51, fév., pp. 77-95.

Bonnet C. et Mahieu C. (2000b). "Public pensions in a dynamic microanalytic framework: the case of France", in Mitton L., Sutherland H., Weeks M. (eds.), Microsimulation Modelling for Policy Analysis, Cambridge University Press.

Bourguignon F., Robilliard A.-S. et Robinson S. (2003). "Representative versus real households in the macro-economic modeling of inequality”, Delta, Document de travail, $\mathrm{n}^{\circ} 2003-05,44 \mathrm{p}$.

Briard K. (2004). « Parcours types des assurés du régime général de la Sécurité Sociale : approche par une classification des carrières », Cerpem, Document de travail, $43 \mathrm{p}$.

Bry X. et Antoine Ph. (2004). «Explorer l'explicatif: application à l'analyse biographique », Ined, Population, vol. 59, $\mathrm{n}^{\circ}$ 6, nov.-déc., pp. 909-946.

Buffeteau S. et Godefroy p. (2005). «Conditions de départ en retraite selon l'âge de fin d'études : analyse prospective pour les générations 1945 à 1974 », Insee, Document de travail, $\mathrm{n}^{\circ} \mathrm{G} 2005 / 01,38 \mathrm{p}$.

Caldwell S. B. (1996). "Health, Wealth, Pensions and Life Paths: The Corsim Dynamic Microsimulation Model", in Harding A. (ed.), Microsimulation and Public Policy, Amsterdam, pp. 505-522. 
Chagny O., Dupont G., Sterdyniak H. et Veroni p. (2001). « Les réformes des systèmes de retraites en Europe $»$, Revue de l'OFCE, n ${ }^{\circ}$ 78, pp. 97-208.

Chambaz Ch. et Le Minez S. (2003). «Analyse des coûts budgétaires, des effets redistributifs et incitatifs des politiques sociales et fiscales affectant le revenu disponible des ménages: l'apport des modèles de microsimulation », Drees, Dossiers Solidarité et Santé, n³, juilletsept., pp. 9-22.

COR - Conseil d'Orientation des Retraites (2002). Retraites : renouveler le contrat social entre les générations, Premier rapport, La Documentation Française, coll. Rapports officiels, 395 p.

COR - Conseil d'Orientation des Retraites (2004). Retraites : les réformes en France et à l'étranger ; le droit à l'information des assurés, Deuxième rapport, La Documentation Française, coll. Rapports officiels, $427 \mathrm{p}$.

Daniel C. (1999). «L’indemnisation du chômage depuis 1979 différenciation des droits, éclatement des statuts », Revue de l'Ires, n 29, 28 p.

Dantec A., Nauze-Fichet E. et Pelgrin F. (2000). "Projection de trajectoires économiques par microsimulation. Quelle équité pour les retraites ?», Revue Économique, vol. 51, hors série, pp. 115-129.

Debrand T., Pennec S. et Privat A.-G. (2004). « Un modèle de microsimulation pour la projection des retraites du régime général », Économie et Prévision, n 160-161, pp. 215-230.

Dupont G., Hagneré C. et Touzé V. (2004). «Les modèles de microsimulation dynamique dans l'analyse des réformes des systèmes de retraites: une tentative de bilan », Économie et Prévision, $\mathrm{n}^{\circ}$ 160-161, pp. 167-191.

Dupuis J.-M. et El Moudden C. (2000). « Carrières atypiques et faibles retraites. Une approche par cas types », Rapport de recherche pour la Drees, Université de Caen.

INSEE (1999). « Le modèle de microsimulation dynamique Destinie », Insee, Document de travail, $\mathrm{n}^{\circ} \mathrm{G} 9913,124 \mathrm{p}$.

Koubi M. (2005). «Élaboration de carrières-types représentatives des carrières complètes des salariés du secteur privé nés en 1948 », Insee, Journées de Modélisation Statistique, Paris, 15 mars.

Lagarde F. (1985). «Une approche longitudinale de la redistribution », in Kessler D. et Masson A. (dir.), Cycles de vie et générations, Economica, pp. 273-284.

Legendre F. (2004). «Micro-simulation et évaluation des politiques économiques et sociales : un panorama des développements récents en France », Revue d'Économie Politique, n 114 , vol. 1, janv-fév, pp. 17-53.

Lucas R. (1976). "Econometric Policy Evaluation: A Critique”, in K. Brunner and A. Meltzer (eds), Carnegie-Rochester Conference Series on Public Policy, vol. 1, pp. 19-46.

Mahieu R. et Sédillot B. (2000). "Microsimulations of the retirement decision: a supply-side approach", Insee, Document de travail, $\mathrm{n}^{\circ} \mathrm{G} 2000 / 07,38 \mathrm{p}$.

Nauze-Fichet E., Lerais F. et Lhermitte S. (2003). «Les projections de population active », Insee, Résultats Société, $\mathrm{n}^{\circ} 13$.

O’Donoghue C. (2001). "Dynamic Microsimulation: A Methodological Survey", Brazilian Electronic Journal of Economics, vol. 4, n 2, $77 \mathrm{p}$. 
OFCE - Timbeau X. et Plane M. (2004). « Projections macroéconomiques pour l'économie française à l'horizon $2050 »$, version provisoire du 5 octobre donnée en séance plénière du Cor du 20 octobre.

Pelé L.-P. et Ralle p. (1999). «Les choix de l'âge de la retraite - aspects incitatifs des règles du régime général et effets de la réforme 1993 », Économie et Prévision, n 138-139, pp. 163-177.

Privat A.-G. (2005). L'avenir des retraites en France-Évaluation de l'impact des réformes de 1993 et de 2003 à l'aide du modèle de microsimulation Artémis, Thèse de Doctorat en Sciences Économiques, Institut d'Études Politiques de Paris, 294 p.

Raynaud E. (2002). «Présentation d'Oscarie: outil de calcul de retraites par cas types », Drees, Dossiers Solidarité et Santé, n 4, pp. 63-69.

Raynaud E. (2004). "Les retraites de la génération 1948, une illustration par quelques cas types », Drees, Études et Résultats, ${ }^{\circ} 331,10$ p.

Robilliard, A.-S., Bourguignon F. et Robinson S. (2002). "Crisis and Income Distribution: A MicroMacro Model for Indonesia”, Banque mondiale, miméo.

Tanchoux J. (1989). Voies de réforme pour les régimes de retraite de base - constitution et utilisation d'un modèle de prévision à vingt ans des charges et ressources du régime général d'assurance vieillesse, Mémoire d'actuariat, Institut de Statistique de l'Université de Paris (Isup), $331 \mathrm{p}$.

Van Imhoff E. et Post W. (1997). «Méthodes de micro-simulation pour des projections de population », Ined, Population, vol. 4, pp. 889-932.

Vernière L. (2001). «Panorama des réformes des systèmes de retraite à l'étranger. Les réformes en Suède et en Italie », CDC, Questions Retraite, $\mathrm{n}^{\circ}$ 2001-38, avril, 24 p.

Walraët E. et Vincent A. (2003). «La redistribution intragénérationnelle dans le système de retraite des salariés du privé : une approche par microsimulation», Insee, Économie et Statistique, pp. 31-56.

\section{Notes}

1. Réunion du Cor du 01/07/2004, «Cadrage et premiers éléments de discussion ».

2. Pour une typologie plus complète des méthodes d'évaluation, $c f$. Dupont et al. (2004).

3. En 2000, l'âge moyen à l'attribution d'un droit direct observé était de 61,7 ans (Cnav, Recueil Statistique 2003, Tab.T4-15).

4. Comme signalé précédemment, on suppose que la proportion globale de départs à taux réduit est constante entre 2003 et 2050. La représentativité du groupe 1 baissant sur la période, la proportion d'assurés liquidant à taux réduit (resp. à taux plein) augmente (resp. baisse) simultanément. 